\title{
Hybrid algorithm with perturbations for total asymptotically non-expansive mappings in CAT(0) space
}

\section{Kenyi Calderón, J. Martínez-Moreno \& E. M. Rojas}

To cite this article: Kenyi Calderón, J. Martínez-Moreno \& E. M. Rojas (2020) Hybrid algorithm with perturbations for total asymptotically non-expansive mappings in CAT(0) space, International Journal of Computer Mathematics, 97:1-2, 405-419, DOI: 10.1080/00207160.2019.1619706

To link to this article: https://doi.org/10.1080/00207160.2019.1619706

Published online: 27 May 2019.

Submit your article to this journal

Џll Article views: 233

Q View related articles 5

View Crossmark data

Citing articles: 1 View citing articles $\sqsubset$ 


\title{
Hybrid algorithm with perturbations for total asymptotically non-expansive mappings in CAT(0) space
}

\author{
Kenyi Calderón (D) a,b , J. Martínez-Moreno (i) ${ }^{b}$ and E. M. Rojas (iD) \\ ${ }^{a}$ Facultad de Ciencias, Universidad de Ciencias Aplicadas y Ambientales, Bogotá, Colombia; ${ }^{b}$ Department of \\ Mathematics, Universidad de Jaén, Jaén, Spain; ' Departamento de Matemáticas, Universidad Nacional de Colombia, \\ Bogotá, Colombia
}

\begin{abstract}
In this paper, we establish strong and $\Delta$-convergence theorems of the modified hybrid-CR three steps iteration with perturbations for total asymptotically non-expansive mapping in CAT(0) spaces. Our results improve and extend the corresponding results from the current literature. We also provide three examples to illustrate the convergence behaviour of the proposed algorithm and numerically compare the convergence of the proposed iteration scheme with the existing schemes.
\end{abstract}

\section{ARTICLE HISTORY}

Received 2 August 2018

Revised 30 March 2019

Accepted 4 April 2019

\section{KEYWORDS}

Fixed point; CAT(0) spaces; three steps iteration; hybrid iteration; perturbations

\section{MATHEMATICS SUBJECT CLASSIFICATIONS 47J25; 47H09}

\section{Introduction and basic definitions}

In a metric space $(X, d)$, a geodesic path joining $x \in X$ and $y \in X$ is a map $c$ from a closed interval $[0, r] \subset \mathbb{R}$ to $X$ such that $c(0)=x, c(r)=y$ and $d(c(t), c(s))=|s-t|$ for all $s, t \in[0, r]$. In particular, the mapping $c$ is an isometry and $d(x, y)=r$. The image of geodesic path (joining $x$ and $y$ ) under $c$ is called a geodesic segment joining $x$ and $y$ which is denoted by $[x, y]$ whenever such a segment exists uniquely. For any $x, y \in X$, we denote the point $z \in[x, y]$ by $z=(1-\alpha) x \oplus \alpha y$, where $0 \leq \alpha \leq 1$ if $d(x, z)=\alpha d(x, y)$ and $d(z, y)=(1-\alpha) d(x, y)$.

A metric space $(X, d)$ is called a geodesic space if any two points of $X$ are joined by a geodesic, and $X$ is said to be uniquely geodesic if there is exactly one geodesic joining $x$ and $y$ for each $x, y \in X$. A subset $C$ of $X$ is called convex if $C$ contains every geodesic segment joining any two points in $C$.

A geodesic triangle $\Delta\left(x_{1}, x_{2}, x_{3}\right)$ in a geodesic metric space $(X, d)$ consists of three points of $X$ (as the vertices of $\Delta$ ) and a geodesic segment between each pair of points (as the edges of $\Delta$ ). A comparison triangle for $\Delta\left(x_{1}, x_{2}, x_{3}\right)$ (denoted by $\bar{\Delta}$ ) is a triangle $\left.\bar{\Delta}\right)\left(x_{1}, x_{2}, x_{3}\right):=\left(\bar{x}_{1}, \bar{x}_{2}, \bar{x}_{3}\right)$ in Euclidean plane $\mathbb{R}^{2}$ such that $d_{\mathbb{R}^{2}}\left(x_{i}, x_{j}\right)=d\left(x_{i}, x_{j}\right)$ for $i, j \in 1,2,3$. A point $x \in\left[x_{1}, x_{2}\right]$ is said to be comparison point for $x \in\left[x_{1}, x_{2}\right]$ if $d\left(x_{1}, x\right)=d_{\mathbb{R}^{2}}\left(x_{1}, x\right)$. The comparison points on $\left[x_{2}, x_{3}\right]$ and $\left[x_{3}, x_{1}\right]$ are defined in same way. Let $\Delta$ be a geodesic triangle in $X$ and $\bar{\Delta}$ its comparison triangle in $\mathbb{R}^{2}$. Then $\Delta$ is said to satisfy $C A T(0)$ inequality if for all $x, y \in \Delta$ and all comparison points $\bar{x}, \bar{y} \in \bar{\Delta}$, $d(x, y) \leq d_{\mathbb{R}^{2}}(x, y)$.

A geodesic metric space $X$ is called a $C A T(0)$ space if all geodesic triangles satisfy the above comparison axiom (i.e. $C A T(0)$ inequality). Some well known examples of $C A T(0)$ spaces are complete 
and simply connected Riemannian manifold having non-positive sectional curvature, pre-Hilbert spaces, R-trees, Euclidean buildings and the complex Hilbert ball with a hyperbolic metric.

Let $x, y \in X$ in a CAT(0) space $X$. For each $t \in[0,1]$, there exists a unique point $z \in[x, y]$ such that

$$
d(x, z)=t d(x, y) \quad \text { and } \quad d(y, z)=(1-t) d(x, y) .
$$

For convenience, from now on, we will use the notation $(1-t) \oplus t y$ for unique point $z$ satisfying (1). For details as regards spaces please see $[9,11,12]$. Some results are recalled here for CAT $(0)$ space $X$.

Lemma 1.1 ([6]): Let $X$ be a CAT(0) space. Then, for all $x, y, z \in X$ and $t \in[0,1]$,

(i) $d((1-t) x \oplus t y, z) \leq(1-t) d(x, z)+t d(y, z)$,

(ii) $d^{2}((1-t) x \oplus y, z) \leq(1-t) d^{2}(x, z)+t d^{2}(y, z)-t(1-t) d^{2}(x, y)$.

Let $x_{i} \in X$ and $\lambda_{i} \in[0,1]$ for $i=1,2, \ldots, n$ such that $\sum_{i=1}^{n} \lambda_{i}=1$. Following the definition of unique point $(1-\alpha) x \oplus \alpha y$ on a geodesic segment $[x, y]$, we build the following notations: $\bigoplus_{i=1}^{2} \lambda_{i} x_{i}=\frac{\lambda_{1}}{\lambda_{1}+\lambda_{2}} x_{1} \oplus \frac{\lambda_{2}}{\lambda_{1}+\lambda_{2}} x_{2}$. By induction, we can write

$$
\bigoplus_{i=1}^{n} \lambda_{i} x_{i}=\left(1-\lambda_{n}\right)\left(\frac{\lambda_{1}}{1-\lambda_{n}} x_{1} \oplus \frac{\lambda_{2}}{1-\lambda_{n}} x_{2} \oplus \cdots \oplus \frac{\lambda_{n-1}}{1-\lambda_{n}} x_{n-1}\right) \oplus \lambda_{n} x_{n} .
$$

In view of Lemma 1.1, we can have the following:

Lemma 1.2 ([20]): Let $X$ be a CAT(0) space with $x, x_{i} \in X$ and $\lambda_{i} \in[0,1]$ for $i=1,2, \ldots, n$ such that $\sum_{i=1}^{n} \lambda_{i}=1$. Then

(i) $d\left(\bigoplus_{i=1}^{n} \lambda_{i} x_{i}, x\right) \leq \sum_{i=1}^{n} d\left(x_{i}, x\right)$;

(ii) $d\left(\bigoplus_{i=1}^{n} \lambda_{i} x_{i}, x\right)^{2} \leq \sum_{i=1}^{n} \lambda_{i} d^{2}\left(x_{i}, x\right)-\lambda_{i} \lambda_{j} d^{2}\left(x_{i}, x_{j}\right)$ for $i, j \in\{1,2, \ldots, n\}$.

Kirk and Panyanak [8] specialized the concept of $\Delta$-convergence to $C A T(0)$ spaces and showed that many Banach space results involving weak convergence have precise analogs in this setting.

Let $\left\{x_{n}\right\}$ be a bounded sequence in a CAT(0) space $X$. For $x \in X$, we set $r\left(x,\left\{x_{n}\right\}\right)=$ $\limsup _{n \rightarrow \infty} d\left(x, x_{n}\right)$. The asymptotic radius $r\left\{x_{n}\right\}$ of $\left\{x_{n}\right\}$ is given by $r\left(\left\{x_{n}\right\}\right)=\inf \left\{r\left(x,\left\{x_{n}\right\}\right): x \in X\right\}$ and the asymptotic center $A\left(\left\{x_{n}\right\}\right)$ of $\left\{x_{n}\right\}$ is the set $A\left(\left\{x_{n}\right\}\right)=\left\{x \in X: r\left(x,\left\{x_{n}\right\}\right)=r\left(\left\{x_{n}\right\}\right)\right\}$.

Lemma 1.3 ([6]): If $\left\{x_{n}\right\}$ is a bounded sequence in a CAT(0) space $X$ with $A\left(\left\{x_{n}\right\}\right)=\{x\}$ and $\left\{u_{n}\right\}$ is a subsequence of $\left\{x_{n}\right\}$ with $A\left(\left\{u_{n}\right\}\right)=\{u\}$ and the sequence $\left\{d\left(x_{n}, u\right)\right\}$ converges, then $x=u$.

Lemma 1.4 ([5]): If $K$ is a closed convex subset of a CAT( 0$)$ space $X$ and if $\left\{x_{n}\right\}$ is a bounded sequence in $K$, then the asymptotic center of $\left\{x_{n}\right\}$ is in $K$.

Lemma 1.5 ([10]): Let $K$ be a closed convex subset of a complete CAT(0) space $X$ and $T: K \longrightarrow K$ be a total asymptotically non-expansive mapping. If $\left\{x_{n}\right\}$ is a bounded sequence in $K \Delta$-converging to $x$ and $\lim _{n \longrightarrow \infty} d\left(x_{n}, T x_{n}\right)=0$, then $x \in K$ and $T x=x$.

Lemma 1.6 ([17]): Suppose that $\left\{a_{n}\right\},\left\{b_{n}\right\}$ and $\left\{\delta_{n}\right\}$ are sequence of non-negative real numbers such that $a_{n+1} \leq\left(1+\delta_{n}\right) a_{n}+b_{n}$ for all $n \geq 1$. If $\sum_{n=1}^{\infty} \delta_{n}<\infty$ and $\sum_{n=1}^{\infty} b_{n}<\infty$, then $\lim _{n \rightarrow \infty} a_{n}$ exists.

Definition $1.1([8])$ : A sequence $\left\{x_{n}\right\}$ in CAT(0) space is said to $\Delta$-convergent to $x \in X$ if $x$ is the unique asymptotic center of $\left\{x_{n}\right\}$ for every subsequence $\left\{u_{n}\right\}$ of $\left\{x_{n}\right\}$. In this case, we write $\Delta-\lim _{n} x_{n}=x$ and call $x$ is the $\Delta$-limit of $\left\{x_{n}\right\}$. 
Definition 1.2 ([7]): Let $K$ be a closed convex subset of a complete CAT(0) space $X$. A bounded sequence $\left\{x_{n}\right\}$ in $K$ is said to converge weakly to $q \in K$ if and only if $\Phi(q)=\inf _{x \in K} \Phi(x)$, where $\Phi(x)=\limsup _{n \rightarrow \infty} d\left(x_{n}, x\right)$.

Let we recall some basics for nonlinear mappings on metric spaces. Let $(X, d)$ be a metric space and $K$ be its non-empty subset. Then $T: K \rightarrow K$ is said a:

(a) Contraction if there exists a $k \in[0,1)$ such that $d(T x, T y) \leq k d(x, y)$, for all $x, y \in K$.

(b) Non-expansive if $d(T x, T y) \leq d(x, y)$, for all $x, y \in K$.

(c) Asymptotically non-expansive if for a sequence $u_{n} \subset[0, \infty)$ with $\lim _{n} \longrightarrow \infty u_{n}=0$ such that $d\left(T^{n} x, T^{n} y\right) \leq\left(1+u_{n}\right) d(x, y)$ for all $x, y \in K$ and $n \geq 1$.

(d) Uniformly L-lipschitzian if there exists $L>0$ such that $d\left(T^{n} x, T^{n} y\right) \leq L d(x, y)$ for all $x, y \in K$ and $n \geq 1$.

(e) Semi-compact if for sequence $\left\{x_{n}\right\}$ in $K$ with $\lim _{n \rightarrow \infty} d\left(x_{n}, T x_{n}\right)=0$, there exists a subsequence $\left\{x_{n_{j}}\right\}$ of $\left\{x_{n}\right\}$ such that $\left\{x_{n_{j}}\right\} \rightarrow p \in K$.

Every contraction mapping in non-expansive and every non-expansive mapping is asymptotically non-expansive mapping. But the converse of each may not be true.

In 2007, Agarwal et al. [1] introduced a new iteration process for non-expansive mappings, which they called 'S-iteration process'. The sequence $\left\{x_{n}\right\}$ in this process is given by

$$
\begin{aligned}
x_{n+1} & =\left(1-\alpha_{n}\right) T x_{n}+\alpha_{n} T y_{n} \\
y_{n} & =\left(1-\beta_{n}\right) x_{n}+\beta_{n} T x_{n} \quad n \geq 1,
\end{aligned}
$$

where $\left\{\alpha_{n}\right\}_{n=1}^{\infty}$ and $\left\{\beta_{n}\right\}_{n=1}^{\infty}$ are real sequences in $[0,1]$. They showed that S-iteration procedure is independent of Ishikawa (and hence of Mann) but converges faster that both of these iterative procedures.

In 2012 R. Chugh et al. [4] introduce the following iterative process:

$$
\begin{aligned}
x_{n+1} & =\left(1-\alpha_{n}\right) y_{n}+\alpha_{n} T y_{n}, \\
y_{n} & =\left(1-\beta_{n}\right) T x_{n}+\beta_{n} T z_{n} \quad n \geq 1, \\
z_{n} & =\left(1-\gamma_{n}\right) x_{n}+\gamma_{n} T x_{n},
\end{aligned}
$$

where $\left\{\alpha_{n}\right\}_{n=1}^{\infty},\left\{\beta_{n}\right\}_{n=1}^{\infty},\left\{\gamma_{n}\right\}_{n=1}^{\infty}$ are real sequences in $[0,1]$. It is called the CR iterative scheme. If we take $\alpha_{n}=0$ the iterative process (3) reduces to S-iteration (2).

On the other hand, Schu [16] defined the modified Mann iterative procedure which is a generalization of Mann iterative procedure. For CAT( 0$)$ spaces it can be written as

$$
x_{n+1}=\left(1-\alpha_{n}\right) x_{n} \oplus \alpha_{n} T^{n} x_{n} \quad n \geq 1,
$$

Tan and $\mathrm{Xu}[18]$ generalized Ishikawa iteration procedure in a similar way.

Chang et al. [3] defined the concept of total asymptotically non-expansive mapping in a framework CAT(0) space as follows:

Definition 1.3: Let $(X, d)$ be a CAT(0) space, $K$ be a non-empty closed convex subset and let $T$ : $K \rightarrow K$ be a mapping. $T$ is said to be total asymptotically non-expansive mapping if there exist nonnegative real sequences $\left\{\mu_{n}\right\},\left\{v_{n}\right\}$, with $\mu_{n} \rightarrow 0, v_{n} \rightarrow 0$ and strictly increasing continuous function $\zeta:[0, \infty) \rightarrow[0, \infty)$ with $\zeta(0)=0$ such that

$$
d\left(T^{n} x, T^{n} y\right) \leq d(x, y)+v_{n} \zeta(d(x, y))+\mu_{n}
$$

for all $x, y \in K$ and $n \geq 1$. 
It follows from the above definitions that each non-expansive mapping is an asymptotically nonexpansive mapping with $k_{n}=1 \forall n>1$ and that each asymptotically non-expansive mapping is a total asymptotically non-expansive mapping with $v_{n}=k_{n}-1, \mu_{n}=0, v_{n} \geq 1, \zeta(t)=t \forall t \geq 0$. Moreover, each asymptotically non-expansive mapping is a uniformly L-Lipschitzian mapping with $L=\sup _{n>1}\left\{k_{n}\right\}$ However, the converse of these statements is not true, in general.

They [3] further studied the iterative approximation of fixed point for total asymptotically nonexpansive mappings using a modified Krasnoselskii-Mann iteration process. Iterative approximation of fixed points of total asymptotically non-expansive mappings has also been studied by $[2,13,14,19]$.

Thakur et al. [19] proposed a modified hybrid-Mann iteration process for iterative approximation of fixed points of total asymptotically non-expansive mappings in CAT( 0$)$ spaces. The sequence $\left\{x_{n}\right\}$ in this iteration is given by

$$
\begin{aligned}
x_{n+1} & =T^{n} y_{n} \\
y_{n} & =\left(1-\alpha_{n}\right) x_{n} \oplus \alpha_{n} T^{n} x_{n} \quad n \geq 1,
\end{aligned}
$$

where $\left\{\alpha_{n}\right\}_{n=1}^{\infty}$ is a real sequence in $[0,1]$.

Pansuwan and Sintunavarat [13], propose a modified hybrid-Ishikawa iteration process as follows:

$$
\begin{aligned}
x_{n+1} & =T^{n} y_{n}, \\
y_{n} & =\left(1-\alpha_{n}\right) z_{n} \oplus \alpha_{n} T^{n} z_{n} \quad n \geq 1, \\
z_{n} & =\left(1-\beta_{n}\right) x_{n} \oplus \beta_{n} T^{n} x_{n},
\end{aligned}
$$

for all $n \in \mathbb{N}$, where $\left\{\alpha_{n}\right\}_{n=1}^{\infty}$ and $\left\{\beta_{n}\right\}_{n=1}^{\infty}$ are real sequences in $[0,1]$.

Motived and inspired by Chugh et al. [4], Kumam et al. [10], Pansuwan and Sintunavarat [13] and Saluja and Postolache [15] and some others, we propose: Consider $K$ to be a non-empty closed convex subset of a complete CAT( 0$)$ space $X$ and $T: K \rightarrow K$ be a total asymptotically non-expansive and uniformly L-lipschitzian mapping with $F(T) \neq \emptyset$. We introduce and study a three-step scheme with perturbations. The scheme is defined as follows:

$$
\begin{aligned}
x_{n+1} & =T^{n}\left(\left(1-\alpha_{n}-\alpha_{n}^{\prime}\right) y_{n} \oplus \alpha_{n} T^{n} y_{n} \oplus \alpha_{n}^{\prime} \epsilon_{n}\right), \\
y_{n} & =\left(1-\beta_{n}-\beta_{n}^{\prime}\right) T^{n} x_{n} \oplus \beta_{n} T^{n} z_{n} \oplus \beta_{n}^{\prime} \epsilon_{n}^{\prime} \quad n \geq 1, \\
z_{n} & =\left(1-\gamma_{n}-\gamma_{n}^{\prime}\right) x_{n} \oplus \gamma_{n} T^{n} x_{n} \oplus \gamma_{n}^{\prime} \epsilon_{n}^{\prime \prime},
\end{aligned}
$$

where $\left\{\epsilon_{n}\right\},\left\{\epsilon_{n}^{\prime}\right\}$ and $\left\{\epsilon_{n}^{\prime \prime}\right\}$ are bounded sequences in $K$ and $\left\{\alpha_{n}\right\}_{n=1}^{\infty},\left\{\beta_{n}\right\}_{n=1}^{\infty},\left\{\gamma_{n}\right\}_{n=1}^{\infty},\left\{\alpha_{n}^{\prime}\right\}_{n=1}^{\infty}$, $\left\{\beta_{n}^{\prime}\right\}_{n=1}^{\infty}$ and $\left\{\gamma_{n}^{\prime}\right\}_{n=1}^{\infty}$ are appropriate real sequences in $[0,1]$.

In this paper, we will establish strong and $\Delta$-convergence theorems of modified three-step sequence with perturbation for total asymptotically non-expansive mapping in the frameworks of CAT(0) spaces. Our results can be particularize to case $\alpha_{n}=0$ and we obtain results for modified hybrid-S-iteration process [10].

\section{Main results}

We will prove the following useful lemmas of proposed iteration with perturbations (8) for a total asymptotically non-expansive mapping in CAT(0) spaces. Moreover, we also prove strong and $\Delta$ convergence theorems under mild conditions.

Lemma 2.1: Let K be a non-empty closed convex subset of a complete CAT(0) space X. Let $T: K \longrightarrow K$ be a total asymptotically non-expansive mapping with $\sum_{n=1}^{\infty} \mu_{n}<\infty, \sum_{n=1}^{\infty} v_{n}<\infty$ and $F(T) \neq \emptyset$. 
Let $\left\{x_{n}\right\}$ be the sequence defined by (8) such that

(C1) $\sum_{n=1}^{\infty} \alpha_{n}^{\prime}<\infty, \sum_{n=1}^{\infty} \beta_{n}^{\prime}<\infty$ and $\sum_{n=1}^{\infty} \gamma_{n}^{\prime}<\infty$.

(C2) There exists a constant $M_{1}>0$ such that $\zeta(r) \leq M_{1} r, r \geq 0$.

Then $\lim _{n \rightarrow \infty} d\left(x_{n}, p\right)$ and $\lim _{n \rightarrow \infty} d\left(x_{n}, F(T)\right)$ exist for all $p \in F(T)$.

Proof: Let $p \in F(T)$. Trivially from (C2), for all $x \in K$,

$$
d\left(T^{n} x, p\right) \leq\left(1+v_{n} M_{1}\right) d(x, p)+\mu_{n} .
$$

From (8), (9) and Lemma 1.1 we have

$$
\begin{aligned}
d\left(z_{n}, p\right) & =d\left(\left(1-\gamma_{n}-\gamma_{n}^{\prime}\right) x_{n} \oplus \gamma_{n} T^{n} x_{n} \oplus \gamma_{n}^{\prime} \epsilon_{n}^{\prime \prime}, p\right) \\
& \leq\left(1-\gamma_{n}-\gamma_{n}^{\prime}\right) d\left(x_{n}, p\right)+\gamma_{n}\left[\left(1+v_{n} M_{1}\right) d\left(x_{n}, p\right)+\mu_{n}\right]+\gamma_{n}^{\prime} d\left(\epsilon_{n}^{\prime \prime}, p\right) \\
& \leq\left(1-\gamma_{n}-\gamma_{n}^{\prime}\right)\left(1+v_{n} M_{1}\right) d\left(x_{n}, p\right)+\gamma_{n}\left[\left(1+v_{n} M_{1}\right) d\left(x_{n}, p\right)+\mu_{n}\right]+\gamma_{n}^{\prime} d\left(\epsilon_{n}^{\prime \prime}, p\right) \\
& =\left(1-\gamma_{n}^{\prime}\right)\left(1+v_{n} M_{1}\right) d\left(x_{n}, p\right)+\gamma_{n} \mu_{n}+\gamma_{n}^{\prime} d\left(\epsilon_{n}^{\prime \prime}, p\right) \\
& \leq\left(1+v_{n} M_{1}\right) d\left(x_{n}, p\right)+\mu_{n}+\gamma_{n}^{\prime} d\left(\epsilon_{n}^{\prime \prime}, p\right) .
\end{aligned}
$$

Again using (8), (10) and Lemma 1.1, we have

$$
\begin{aligned}
d\left(y_{n}, p\right)= & d\left(\left(1-\beta_{n}-\beta_{n}^{\prime}\right) T^{n} x_{n} \oplus \beta_{n} T^{n} z_{n} \oplus \beta_{n}^{\prime} \epsilon_{n}^{\prime}, p\right) \\
\leq & \left(1-\beta_{n}-\beta_{n}^{\prime}\right)\left[\left(1+v_{n} M_{1}\right) d\left(x_{n}, p\right)+\mu_{n}\right] \\
& +\beta_{n}\left[\left(1+v_{n} M_{1}\right) d\left(z_{n}, p\right)+\mu_{n}\right]+\beta_{n}^{\prime} d\left(\epsilon_{n}^{\prime}, p\right) \\
\leq & \left(1-\beta_{n}-\beta_{n}^{\prime}\right)\left[\left(1+v_{n} M_{1}\right) d\left(x_{n}, p\right)+\mu_{n}\right] \\
& +\beta_{n}\left[\left(1+v_{n} M_{1}\right)\left[\left(1+v_{n} M_{1}\right) d\left(x_{n}, p\right)+\mu_{n}+\gamma_{n}^{\prime} d\left(\epsilon_{n}^{\prime \prime}, p\right)\right]+\mu_{n}\right] \\
& +\beta_{n}^{\prime} d\left(\epsilon_{n}^{\prime}, p\right)
\end{aligned}
$$

i.e.

$$
\begin{aligned}
d\left(y_{n}, p\right) \leq & \left(1-\beta_{n}-\beta_{n}^{\prime}\right)\left(1+v_{n} M_{1}\right)^{2} d\left(x_{n}, p\right)+\left(1+v_{n} M_{1}\right)\left(1-\beta_{n}-\beta_{n}^{\prime}\right) \mu_{n} \\
& +\beta_{n}\left(1+v_{n} M_{1}\right)^{2} d\left(x_{n}, p\right)+\beta_{n} \mu_{n}\left(1+v_{n} M_{1}\right)+\beta_{n} \mu_{n} \\
& +\beta_{n} \gamma_{n}^{\prime}\left(1+v_{n} M_{1}\right) d\left(\epsilon_{n}^{\prime \prime}, p\right)+\beta_{n}^{\prime} d\left(\epsilon_{n}^{\prime}, p\right) \\
= & \left(1-\beta_{n}^{\prime}\right)\left(1+v_{n} M_{1}\right)^{2} d\left(x_{n}, p\right)+\left(1+v_{n} M_{1}\right)\left(1-\beta_{n}^{\prime}\right) \mu \\
& +\beta_{n} \mu_{n}+\beta_{n}\left(1+v_{n} M_{1}\right) \gamma_{n}^{\prime} d\left(\epsilon_{n}^{\prime \prime}, p\right)+\beta_{n}^{\prime} d\left(\epsilon_{n}^{\prime}, p\right) \\
\leq & \left(1+v_{n} M_{1}\right)^{2} d\left(x_{n}, p\right)+\left(2+v_{n} M_{1}\right) \mu_{n}+\left(1+v_{n} M_{1}\right) \gamma_{n}^{\prime} d\left(\epsilon_{n}^{\prime \prime}, p\right)+\beta_{n}^{\prime} d\left(\epsilon_{n}^{\prime}, p\right) .
\end{aligned}
$$

Set $w_{n}=\left(1-\alpha_{n}-\alpha_{n}^{\prime}\right) y_{n} \oplus \alpha_{n} T^{n} y_{n} \oplus \alpha_{n}^{\prime} \epsilon_{n}$. Now using (12) and Lemma 1.1, we get

$$
\begin{aligned}
d\left(w_{n}, p\right)= & d\left(\left(1-\alpha_{n}-\alpha_{n}^{\prime}\right) y_{n} \oplus \alpha_{n} T^{n} y_{n} \oplus \alpha_{n}^{\prime} \epsilon_{n}, p\right) \\
\leq & \left(1-\alpha_{n}^{\prime}+\alpha_{n} v_{n} M_{1}\right)\left[\left(1+v_{n} M_{1}\right)^{2} d\left(x_{n}, p\right)+\left(2+v_{n} M_{1}\right) \mu_{n}\right. \\
& \left.+\left(1+v_{n} M_{1}\right) \gamma_{n}^{\prime} d\left(\epsilon_{n}^{\prime \prime}, p\right)+\beta_{n}^{\prime} d\left(\epsilon_{n}^{\prime}, p\right)\right]+\alpha_{n} \mu_{n}+\alpha_{n}^{\prime} d\left(\epsilon_{n}, p\right) \\
\leq & \left(1+v_{n} M_{1}\right)^{3} d\left(x_{n}, p\right)+\left[\left(1+v_{n} M_{1}\right)\left(2+v_{n} M_{1}\right)+\alpha_{n}\right] \mu_{n} \\
& +\left(1+v_{n} M_{1}\right)^{2} \gamma_{n}^{\prime} d\left(\epsilon_{n}^{\prime \prime}, p\right)+\left(1+v_{n} M_{1}\right) \beta_{n}^{\prime} d\left(\epsilon_{n}^{\prime}, p\right)+\alpha_{n}^{\prime} d\left(\epsilon_{n}, p\right) .
\end{aligned}
$$


From (5), (8) and (13) we have

$$
d\left(x_{n+1}, p\right)=d\left(T^{n}\left(w_{n}\right), p\right) \leq\left(1+v_{n} M_{1}\right) d\left(w_{n}, p\right)+\mu_{n} \leq\left(1+A_{n}\right) d\left(x_{n}, p\right)+B_{n}+\varepsilon_{n},
$$

where $A_{n}=4 M_{1} v_{n}+6 M_{1}^{2} v_{n}^{2}+4 M_{1}^{3} v_{n}^{3}+M_{1}^{4} v_{n}^{4}, \quad B_{n}=\left(3+5 v_{n} M_{1}+4 v_{n}^{2} M_{1}^{2}+v_{n}^{3} M_{1}^{3}+\left(1+v_{n}\right.\right.$ $\left.\left.M_{1}\right)\right) \mu_{n}$ and $\varepsilon_{n}=\left(1+v_{n} M_{1}\right)^{3} \gamma_{n}^{\prime} d\left(\epsilon_{n}^{\prime \prime}, p\right)+\left(1+v_{n} M_{1}\right)^{2} \beta_{n}^{\prime} d\left(\epsilon_{n}^{\prime}, p\right)+\left(1+v_{n} M_{1}\right) \alpha_{n}^{\prime} d\left(\epsilon_{n}, p\right)$. Since $\left\{\epsilon_{n}\right\},\left\{\epsilon_{n}^{\prime}\right\}$ and $\left\{\epsilon_{n}^{\prime \prime}\right\}$ are bounded sequences in $K$, we can put

$$
M_{2}=\sup _{n \geq 1, x \in K}\left\{d\left(\epsilon_{n}, x\right), d\left(\epsilon_{n}^{\prime}, x\right), d\left(\epsilon_{n}^{\prime \prime}, x\right)\right\} .
$$

Then $M_{2}$ is a finite number and there exists some positive constant $D$ such that $\varepsilon_{n} \leq D\left(\gamma_{n}^{\prime}+\beta_{n}^{\prime}+\right.$ $\alpha_{n}^{\prime}$ ). Taking infimum over all $p \in F(T)$, we get

$$
d\left(x_{n+1}, p\right) \leq\left(1+A_{n}\right) d\left(x_{n}, F(T)\right)+B_{n}+D\left(\gamma_{n}^{\prime}+\beta_{n}^{\prime}+\alpha_{n}^{\prime}\right) .
$$

Since $\sum_{n=1}^{\infty} \mu_{n}<\infty, \sum_{n=1}^{\infty} v_{n}<\infty$, it follows that $\sum_{n=1}^{\infty} A_{n}<\infty, \sum_{n=1}^{\infty} B_{n}<\infty$. From (C1) and Lemma 1.6, $\lim _{n \rightarrow \infty} d\left(x_{n}, p\right)$ and $\lim _{n \rightarrow \infty} d\left(x_{n}, F(T)\right)$ exist.

Theorem 2.1: Let $X, K, T,\left\{x_{n}\right\}$ satisfy the hypothesis of Lemma 2.1. Then the sequence $\left\{x_{n}\right\}$ defined by (8) converges strongly to a fixed point of $T$ if and only if $\lim _{n \rightarrow \infty} d\left(x_{n}, F(T)\right)=0$, where $d(x, F(T))=\inf \{d(x, p): p \in F(T)\}$.

Proof: The necessity is obvious. To prove the converse, suppose that $\lim _{\inf } \operatorname{si\infty }_{n \rightarrow \infty} d\left(x_{n}, F(T)\right)=0$. Thus by hypothesis $\lim _{n \rightarrow \infty} d\left(x_{n}, F(T)\right)=0$. Next, we show that $\left\{x_{n}\right\}$ is Cauchy sequence in $K$. with help of inequality $1+x \leq e^{x}, x \geq 0$. From Lemma $2.1, d\left(x_{n+1}, p\right) \leq\left(1+A_{n}\right) d\left(x_{n}, p\right)+B_{n}+\varepsilon_{n}$, i.e, for any integer $m \geq 1$,

$$
\begin{aligned}
& d\left(x_{n+m}, p\right) \leq\left(1+A_{n+m-1}\right) d\left(x_{n+m-1}, p\right)+B_{n+m-1}+\varepsilon_{n+m-1} \\
& \leq e^{A_{n+m-1}} d\left(x_{n+m-1}, p\right)+B_{n+m-1}+\varepsilon_{n+m-1} \\
& \leq e^{A_{n+m-1}}\left[e^{A_{n+m-2}} d\left(x_{n+m-2}, p\right)+B_{n+m-2}+\varepsilon_{n+m-2}\right] \\
& +B_{n+m-1}+\varepsilon_{n+m-1} \\
& \leq \cdots \\
& \leq\left(e^{\sum_{k=n}^{\infty} A_{k}}\right) d\left(x_{n}, p\right)+\left(e^{\sum_{k=n}^{\infty} A_{k}}\right) \sum_{k=n}^{n+m-1} B_{k} \\
& +\left(e^{\sum_{k=n}^{\infty} A_{k}}\right) \sum_{k=n}^{n+m-1} \varepsilon_{k} \\
& =G d\left(x_{n}, p\right)+G \sum_{k=n}^{n+m-1} B_{k}+G \sum_{k=n}^{n+m-1} \varepsilon_{k} \text {, }
\end{aligned}
$$

where $G=e^{\sum_{k=n}^{\infty} A_{k}}$.

Since $\lim _{n \rightarrow \infty} d\left(x_{n}, F(T)\right)=0$, without loss of generality, we may assume that a subsequence $\left\{x_{n_{k}}\right\}$ of $\left\{x_{n}\right\}$ and subsequence $\left\{p_{n_{k}}\right\} \subset F(T)$ such that $d\left(x_{n_{k}}, p_{n_{k}}\right) \rightarrow 0$ as $k \rightarrow \infty$. Then for any $\epsilon>0$, there exists $k_{\epsilon}>0$ such that

$$
d\left(x_{n_{k}}, p_{n_{k}}\right)<\frac{\epsilon}{6 G}, \quad \sum_{k=n}^{\infty} B_{k}<\frac{\epsilon}{6 G} \quad \text { and } \quad \sum_{k=n}^{\infty} \varepsilon_{k}<\frac{\epsilon}{6 G}
$$

for all $k \geq k_{\epsilon}$. 
For any $m \geq 1$ and for all $n \geq n_{k}$, by (14), we have

$$
\begin{aligned}
d\left(x_{n+m}, x_{n}\right) \leq & d\left(x_{n+m}, p_{n k}\right)+d\left(x_{n}, p_{n k}\right) \\
\leq & G d\left(x_{n}, p_{n k}\right)+G \sum_{k=n_{k_{\epsilon}}}^{\infty} B_{k}+G \sum_{k=n}^{\infty} \varepsilon_{k} \\
& +G d\left(x_{n}, p_{n k}\right)+G \sum_{k=n_{k_{\epsilon}}}^{\infty} B_{k}+G \sum_{k=n}^{\infty} \varepsilon_{k} \\
\leq & 2 G d\left(x_{n}, p_{n k}\right)+2 G \sum_{k=n_{k \epsilon}}^{\infty} B_{k}+2 G \sum_{k=n}^{\infty} \varepsilon_{k} \\
\leq & 2 G \frac{\epsilon}{6 G}+2 G \frac{\epsilon}{6 G}+2 G \frac{\epsilon}{6 G}=\epsilon .
\end{aligned}
$$

This proves that $\left\{x_{n}\right\}$ is a Cauchy sequence in $K$. Thus, the completeness of $X$ implies that $\left\{x_{n}\right\}$ must be convergence. Assume that $\lim _{n \rightarrow \infty} x_{n}=q$. Since $K$ is closed, therefore $q \in K$. Next, we show that $q \in F(T)$. Since $\lim _{n \rightarrow \infty} d\left(x_{n}, F(T)\right)=0$, we get $d(q, F(T))=0$, closedness of $F(T)$ gives that $q \in$ $F(T)$. Thus $\left\{x_{n}\right\}$ converges strongly to a point in $F(T)$. This completes the proof.

Lemma 2.2: Let $K$ be a non-empty closed convex subset of a complete CAT(0) space X. Let $T: K \longrightarrow K$ be a uniformly continuous and total asymptotically non-expansive mapping with $\sum_{n=1}^{\infty} \mu_{n}<\infty, \sum_{n=1}^{\infty} v_{n}<\infty$ and $F(T) \neq \emptyset$. Let $\left\{\alpha_{n}\right\},\left\{\beta_{n}\right\}$ and $\left\{\gamma_{n}\right\}$ be sequences in $(0,1)$ such that, $\liminf _{n \rightarrow \infty} \alpha_{n}\left(1-\alpha_{n}-\alpha_{n}^{\prime}\right)>0, \liminf _{n \rightarrow \infty} \beta_{n}\left(1-\beta_{n}-\beta_{n}^{\prime}\right)>0$ and $\liminf _{n \rightarrow \infty} \gamma_{n}\left(1-\gamma_{n}-\right.$ $\left.\gamma_{n}^{\prime}\right)>0$. Let $\left\{x_{n}\right\}$ be the sequence defined by (8) such that

(C1) $\sum_{n=1}^{\infty} \alpha_{n}^{\prime}<\infty, \sum_{n=1}^{\infty} \beta_{n}^{\prime}<\infty$ and $\sum_{n=1}^{\infty} \gamma_{n}^{\prime}<\infty$.

(C2) There exists a constant $M_{1}>0$ such that $\zeta(r) \leq M_{1} r, r \geq 0$.

Then $\lim _{n \rightarrow \infty} d\left(T x_{n}, x_{n}\right)=0$.

Proof: For any $p \in F(T)$, it follows from Lemma 2.1 that $\lim _{n \rightarrow \infty} d\left(x_{n}, p\right)$ exists. Let $\lim _{n \rightarrow \infty} d\left(x_{n}, p\right)$ $=q$, for some $q \geq 0$. We claim that $\lim _{n \rightarrow \infty} d\left(T x_{n}, x_{n}\right)=0$.

Since $\left\{x_{n}\right\}$ is bounded, there exists $R>0$ such that $\left\{x_{n}\right\},\left\{y_{n}\right\},\left\{z_{n}\right\} \subset B_{R}(p)$ for all $n \geq 1$ Using (8) and Lemma 1.2,

$$
\begin{aligned}
d^{2}\left(z_{n}, p\right)=d^{2} & \left(\left(1-\gamma_{n}-\gamma_{n}^{\prime}\right) x_{n} \oplus \gamma_{n} T^{n} x_{n} \oplus \gamma_{n}^{\prime} \epsilon_{n}^{\prime \prime}, p\right) \\
\leq & \gamma_{n} d^{2}\left(T^{n} x_{n}, p\right)+\left(1-\gamma_{n}-\gamma_{n}^{\prime}\right) d^{2}\left(x_{n}, p\right)+\gamma_{n}^{\prime} d^{2}\left(\epsilon_{n}^{\prime \prime}, p\right) \\
& -\gamma_{n}\left(1-\gamma_{n}-\gamma_{n}^{\prime}\right) d^{2}\left(T^{n} x_{n}, x_{n}\right) \\
\leq & \gamma_{n}\left[\left(1+v_{n} M_{1}\right) d\left(x_{n}, p\right)+\mu_{n}\right]^{2}+\left(1-\gamma_{n}-\gamma_{n}^{\prime}\right) d^{2}\left(x_{n}, p\right)+\gamma_{n}^{\prime} d^{2}\left(\epsilon_{n}^{\prime \prime}, p\right) \\
& -\gamma_{n}\left(1-\gamma_{n}-\gamma_{n}^{\prime}\right) d^{2}\left(T^{n} x_{n}, x_{n}\right) \\
\leq & \gamma_{n}\left(1+v_{n} M_{1}\right)^{2} d^{2}\left(x_{n}, p\right)+\gamma_{n} \mu_{n}\left(2\left(1+v_{n} M_{1}\right) d\left(x_{n}, p\right)+\mu_{n}\right) \\
& +\left(1-\gamma_{n}-\gamma_{n}^{\prime}\right)\left(1+v_{n} M_{1}\right)^{2} d^{2}\left(x_{n}, p\right)+\gamma_{n}^{\prime} d^{2}\left(\epsilon_{n}^{\prime \prime}, p\right) \\
& -\gamma_{n}\left(1-\gamma_{n}-\gamma_{n}^{\prime}\right) d^{2}\left(T^{n} x_{n}, x_{n}\right)
\end{aligned}
$$




$$
\begin{aligned}
= & \left(1-\gamma_{n}^{\prime}\right)\left(1+v_{n} M_{1}\right)^{2} d^{2}\left(x_{n}, p\right)+\gamma_{n} \mu_{n}\left(2\left(1+v_{n} M_{1}\right) d\left(x_{n}, p\right)+\mu_{n}\right)+\gamma_{n}^{\prime} d^{2}\left(\epsilon_{n}^{\prime \prime}, p\right) \\
& -\gamma_{n}\left(1-\gamma_{n}-\gamma_{n}^{\prime}\right) d^{2}\left(T^{n} x_{n}, x_{n}\right) \\
\leq & d^{2}\left(x_{n}, p\right)+P_{n} v_{n}+Q_{n} \mu_{n}+\gamma_{n}^{\prime} d^{2}\left(\epsilon_{n}^{\prime \prime}, p\right)-\gamma_{n}\left(1-\gamma_{n}-\gamma_{n}^{\prime}\right) d^{2}\left(T^{n} x_{n}, x_{n}\right)
\end{aligned}
$$

where $P_{n}=M_{1}\left(2+v_{n} M_{1}\right) d^{2}\left(x_{n}, p\right)$ and $Q_{n}=2\left(1+v_{n} M_{1}\right) d\left(x_{n}, p\right)+\mu_{n}$. This implies that

$$
d^{2}\left(z_{n}, p\right) \leq d^{2}\left(x_{n}, p\right)+P_{n} v_{n}+Q_{n} \mu_{n}+\gamma_{n}^{\prime} d^{2}\left(\epsilon_{n}^{\prime \prime}, p\right)
$$

From (17) we have,

$$
\gamma_{n}\left(1-\gamma_{n}-\gamma_{n}^{\prime}\right) d^{2}\left(T^{n} x_{n}, x_{n}\right) \leq d^{2}\left(x_{n}, p\right)+P_{n} v_{n}+Q_{n} \mu_{n}-d^{2}\left(z_{n}, p\right)+\gamma_{n}^{\prime} M_{2}
$$

Since $\sum_{n=1}^{\infty} \mu_{n}<\infty, \sum_{n=1}^{\infty} v_{n}<\infty, \sum_{n=1}^{\infty} \gamma_{n}^{\prime}<\infty$ and $d\left(x_{n}, p\right) \leq R$ for all $n$, we have $\gamma_{n}\left(1-\gamma_{n}-\right.$ $\left.\gamma_{n}^{\prime}\right) d^{2}\left(T^{n} x_{n}, x_{n}\right)<\infty$. By $\lim \inf _{n \rightarrow \infty} \gamma_{n}\left(1-\gamma_{n}-\gamma_{n}^{\prime}\right)>0$, it follows that

$$
\lim _{n \rightarrow \infty} d\left(T^{n} x_{n}, x_{n}\right)=0
$$

Again from (8) and Lemma 1.1, we have

$$
\begin{aligned}
d^{2}\left(y_{n}, p\right) \leq & d^{2}\left(\left(1-\beta_{n}-\beta_{n}^{\prime}\right) T^{n} x_{n} \oplus \beta_{n} T^{n} z_{n} \oplus \beta_{n}^{\prime} \epsilon_{n}^{\prime}, p\right) \\
\leq & \beta_{n} d^{2}\left(T^{n} z_{n}, p\right)+\left(1-\beta_{n}-\beta_{n}^{\prime}\right) d^{2}\left(T^{n} x_{n}, p\right)+\beta_{n}^{\prime} d^{2}\left(\epsilon_{n}^{\prime}, p\right) \\
& -\beta_{n}\left(1-\beta_{n}-\beta_{n}^{\prime}\right) d^{2}\left(T^{n} x_{n}, T^{n} z_{n}\right) \\
\leq & \beta_{n}\left[\left(1+v_{n} M_{1}\right) d\left(z_{n}, p\right)+\mu_{n}\right]^{2} \\
& +\left(1-\beta_{n}-\beta_{n}^{\prime}\right)\left[\left(1+v_{n} M_{1}\right) d\left(x_{n}, p\right)+\mu_{n}\right]^{2}+\beta_{n}^{\prime} d^{2}\left(\epsilon_{n}^{\prime}, p\right) \\
& -\beta_{n}\left(1-\beta_{n}-\beta_{n}^{\prime}\right) d^{2}\left(T^{n} x_{n}, T^{n} z_{n}\right) \\
= & \beta_{n}\left(1+v_{n} M_{1}\right)^{2} d^{2}\left(z_{n}, p\right)+\beta_{n}\left[2 \mu_{n} d\left(z_{n}, p\right)+\mu_{n}^{2}\right] \\
& +\left(1-\beta_{n}-\beta_{n}^{\prime}\right)\left[\left(1+v_{n} M_{1}\right)^{2} d^{2}\left(x_{n}, p\right)+Q_{n}^{\prime} \mu_{n}+\mu_{n}^{2}\right] \\
& +\beta_{n}^{\prime} d^{2}\left(\epsilon_{n}^{\prime}, p\right)-\beta_{n}\left(1-\beta_{n}-\beta_{n}^{\prime}\right) d^{2}\left(T^{n} x_{n}, T^{n} z_{n}\right),
\end{aligned}
$$

where $Q_{n}^{\prime}=Q_{n}-\mu_{n}$.

Substituting (18) into (20), we have

$$
\begin{aligned}
d^{2}\left(y_{n}, p\right) \leq & \beta_{n}\left(1+v_{n} M_{1}\right)^{2}\left[d^{2}\left(x_{n}, p\right)+P_{n} v_{n}+Q_{n}^{\prime} \mu_{n}+\gamma_{n}^{\prime} d^{2}\left(\epsilon_{n}^{\prime \prime}, p\right)\right] \\
& +\beta_{n}\left[2 \mu_{n} d\left(z_{n}, p\right)+\mu_{n}^{2}\right] \\
& +\left(1-\beta_{n}-\beta_{n}^{\prime}\right)\left[\left(1+v_{n} M_{1}\right)^{2} d^{2}\left(x_{n}, p\right)+Q_{n}^{\prime} \mu_{n}+\mu_{n}^{2}\right] \\
& +\beta_{n}^{\prime} d^{2}\left(\epsilon_{n}^{\prime}, p\right)-\beta_{n}\left(1-\beta_{n}-\beta_{n}^{\prime}\right) d^{2}\left(T^{n} x_{n}, T^{n} z_{n}\right) \\
\leq & \left(1+v_{n} M_{1}\right)^{2} d^{2}\left(x_{n}, p\right)
\end{aligned}
$$




$$
\begin{aligned}
& +\left(1+v_{n} M_{1}\right)^{2}\left[P_{n} v_{n}+Q_{n}^{\prime} \mu_{n}+\gamma_{n}^{\prime} d^{2}\left(\epsilon_{n}^{\prime \prime}, p\right)\right] \\
& +2 \mu_{n} d\left(z_{n}, p\right)+Q_{n}^{\prime} \mu_{n}+\mu_{n}^{2} \\
& +\beta_{n}^{\prime} d^{2}\left(\epsilon_{n}^{\prime}, p\right)-\beta_{n}\left(1-\beta_{n}-\beta_{n}^{\prime}\right) d^{2}\left(T^{n} x_{n}, T^{n} z_{n}\right) \\
= & \left(1+v_{n} M_{1}\right)^{2} d^{2}\left(x_{n}, p\right)+\left(1+v_{n} M_{1}\right)^{2} P_{n} v_{n} \\
& +\left(1+v_{n} M_{1}\right)^{2} Q_{n}^{\prime} \mu_{n}+2 \mu_{n} d\left(z_{n}, p\right)+Q_{n}^{\prime} \mu_{n}+\mu_{n}^{2} \\
& +\gamma_{n}^{\prime}\left(1+v_{n} M_{1}\right)^{2} d^{2}\left(\epsilon_{n}^{\prime \prime}, p\right)+\beta_{n}^{\prime} d^{2}\left(\epsilon_{n}^{\prime}, p\right) \\
& -\beta_{n}\left(1-\beta_{n}-\beta_{n}^{\prime}\right) d^{2}\left(T^{n} x_{n}, T^{n} z_{n}\right) \\
\leq & d^{2}\left(x_{n}, p\right)+R_{n} v_{n}+S_{n} \mu_{n} \\
& +\gamma_{n}^{\prime}\left(1+v_{n} M_{1}\right)^{2} d^{2}\left(\epsilon_{n}^{\prime \prime}, p\right)+\beta_{n}^{\prime} d^{2}\left(\epsilon_{n}^{\prime}, p\right) \\
& -\beta_{n}\left(1-\beta_{n}-\beta_{n}^{\prime}\right) d^{2}\left(T^{n} x_{n}, T^{n} z_{n}\right),
\end{aligned}
$$

where $R_{n}=P_{n}+\left(1+v_{n} M_{1}\right)^{2} P_{n}$ and $S_{n}=Q_{n}+\left(1+v_{n} M_{1}\right)^{2} Q_{n}^{\prime}+2 d\left(z_{n}, p\right)$. This implies that

$$
\beta_{n}\left(1-\beta_{n}-\beta_{n}^{\prime}\right) d^{2}\left(T^{n} x_{n}, T^{n} z_{n}\right) \leq d^{2}\left(x_{n}, p\right)-d^{2}\left(y_{n}, p\right)+R_{n} v_{n}+S_{n} \mu_{n}+\lambda_{n} M_{2},
$$

where $\lambda_{n}=\beta_{n}^{\prime}+\gamma_{n}^{\prime}\left(1+v_{n} M_{1}\right)^{2}$.

Since $\sum_{n=1}^{\infty} \mu_{n}<\infty, \sum_{n=1}^{\infty} v_{n}<\infty, \sum_{n=1}^{\infty} \beta_{n}^{\prime}<\infty, \sum_{n=1}^{\infty} \gamma_{n}^{\prime}<\infty$ and $d\left(x_{n}, p\right) \leq R$ and $d\left(z_{n}, p\right) \leq R$ for all $n$, we have $\beta_{n}\left(1-\beta_{n}-\beta_{n}^{\prime}\right) d^{2}\left(T^{n} x_{n}, T^{n} z_{n}\right)<\infty$. This implies by lim inf $\lim _{n \rightarrow \infty} \beta_{n}$ $\left(1-\beta_{n}-\beta_{n}^{\prime}\right)>0$ that

$$
\lim _{n \rightarrow \infty} d\left(T^{n} x_{n}, T^{n} z_{n}\right)=0 .
$$

In a similar way, we can obtain that

$$
\lim _{n \rightarrow \infty} d\left(T^{n} y_{n}, y_{n}\right)=0
$$

By using (5) and (8), we get

$$
\begin{aligned}
d\left(T^{n} y_{n}, x_{n+1}\right) & =d\left(T^{n} y_{n}, T^{n} w_{n}\right) \leq\left(1+v_{n} M_{1}\right) d\left(y_{n}, w_{n}\right)+\mu_{n} \\
& \leq\left(1+v_{n} M_{1}\right)\left[\alpha_{n} d\left(y_{n}, T^{n} y_{n}\right)+\alpha_{n}^{\prime} d\left(y_{n}, \epsilon_{n}\right)\right]+\mu_{n} .
\end{aligned}
$$

From (23), we get $\lim _{n \rightarrow \infty} d\left(T^{n} y_{n}, x_{n+1}\right)=0$. Note that

$$
\begin{aligned}
d\left(y_{n}, T^{n} x_{n}\right) & \leq d\left(\left(1-\beta_{n}-\beta_{n}^{\prime}\right) T^{n} x_{n} \oplus \beta_{n} T^{n} z_{n} \oplus \beta_{n}^{\prime} \epsilon_{n}^{\prime}, T^{n} x_{n}\right) \\
& \leq \beta_{n} d\left(T^{n} z_{n} T^{n} x_{n}\right)+\beta_{n}^{\prime} M_{2} \rightarrow 0, \quad \text { as } n \rightarrow \infty .
\end{aligned}
$$

By (19) and (25), we have $d\left(x_{n}, y_{n}\right) \leq d\left(x_{n}, T^{n} x_{n}\right)+d\left(T^{n} x_{n}, y_{n}\right) \rightarrow 0$ as $n \rightarrow \infty$. By triangular inequality, $d\left(x_{n}, x_{n+1}\right) \leq d\left(x_{n}, y_{n}\right)+d\left(y_{n}, T^{n} y_{n}\right)+d\left(T^{n} y_{n}, x_{n+1}\right) \rightarrow 0$, as $n \rightarrow \infty$. By (19) and uniform continuity of $T$, we have

$$
\begin{aligned}
d\left(x_{n}, T x_{n}\right) \leq & d\left(x_{n}, x_{n+1}\right)+d\left(x_{n+1}, T^{n+1} x_{n+1}\right) \\
& +d\left(T^{n+1} x_{n+1}, T^{n+1} x_{n}\right)+d\left(T^{n+1} x_{n}, T x_{n}\right) \\
\leq & \left(2+v_{n+1} M_{1}\right) d\left(x_{n+1}, x_{n}\right)+d\left(x_{n+1}, T^{n+1} x_{n+1}\right) \\
& +d\left(T^{n+1} x_{n}, T x_{n}\right)+\mu_{n+1} \\
& \rightarrow 0, \quad \text { as } n \rightarrow \infty .
\end{aligned}
$$

This completes the proof. 
Theorem 2.2: Let $X, K, T,\left\{x_{n}\right\}$ satisfy the hypothesis of Lemma 2.2. Then the sequence $\left\{x_{n}\right\}$ defined by (8) $\Delta$-converges to a fixed point of $T$.

Proof: We first show that $w_{\Delta}\left(\left\{x_{n}\right\}\right) \subseteq F(T)$. We denote $w_{\Delta}\left(\left\{x_{n}\right\}\right):=\cup\left(A\left\{u_{n}\right\}\right)$ where the union is taken over all subsequence $\left\{u_{n}\right\}$ of $\left\{x_{n}\right\}$. Let $u \in w_{\Delta}\left(\left\{x_{n}\right\}\right)$ then there exists a subsequence $\left\{u_{n}\right\}$ of $\left\{x_{n}\right\}$ such that $A\left(\left\{u_{n}\right\}\right)=\{u\}$. By Lemma 1.4 there exists a subsequence $\left\{v_{n}\right\}$ of $\left\{u_{n}\right\}$ such that $\Delta-$ $\lim _{n} v_{n}=v \in K$. By Lemma $1.5,\left\{v_{n}\right\} \in F(T)$. Now by Lemma $2.1 \lim _{n} \longrightarrow \infty d\left(x_{n}, F(T)\right)$ exists, so by Lemma 1.3, we have $u=v$, i.e, $w_{\Delta}\left(\left\{x_{n}\right\}\right) \subseteq F(T)$.

To show that $\left\{x_{n}\right\} \Delta-$ convergent to point in $F(T)$, it is sufficient to show that $w_{\Delta}\left(\left\{x_{n}\right\}\right)$ consists of exactly one point.

Let $\left\{u_{n}\right\}$ be subsequence of $\left\{x_{n}\right\}$ with $A\left(\left\{u_{n}\right\}\right)=\{u\}$, and let $A\left(\left\{x_{n}\right\}\right)=\{x\}$ for some $u \in$ $w_{\Delta}\left(\left\{x_{n}\right\}\right) \subseteq F(T)$ and $\left\{d\left(x_{n}, v\right)\right\}$ converge. By Lemma 1.3, we have $x=v \in F(T)$. Thus $w_{\Delta}\left(\left\{x_{n}\right\}\right)=$ $\{x\}$. This shows that $\left\{x_{n}\right\}$ is $\Delta-$ convergent to a point of $F(T)$.

Theorem 2.3: Let $X, K, T,\left\{x_{n}\right\}$ satisfy the hypothesis of Lemma 2.2. If $T^{m}$ is semi-compact for some $m \in \mathbb{N}$, then the sequence $\left\{x_{n}\right\}$ converges strongly to a point of $F(T)$.

Proof: By Lemma 2.2 and the uniformly continuity of $T$, we have $\lim _{n \rightarrow \infty} d\left(x_{n}, T^{m} x_{n}\right)=0$. By the semi-compactness of $T^{m}$, there exists a subsequence $\left\{x_{n_{j}}\right\}$ of $\left\{x_{n}\right\}$ such that $x_{n_{j}} \rightarrow p \in K$. Lemma 2.2 guarantees that $\lim _{n_{j} \longrightarrow \infty} d\left(x_{n_{j}}, T x_{n_{j}}\right)=0$ and so $d(p, T p)=0$. By Lemma $2.1, \lim _{n \rightarrow \infty} d\left(x_{n}, p\right)$, thus $p$ is the strong limit of the sequence $\left\{x_{n}\right\}$ itself.

\section{Numerical examples}

In this section, we provide the numerical examples to illustrate its performance and to compare propose iteration with existing methods.

Example 3.1: Let $X:=\mathbb{R}$ be a usual metric space with the metric $d$, which is also a complete CAT(0) space, and $C=[1,999]$. We see that $C$ is a bounded closed convex subset of $X$. Define a mapping

$$
T x=\sqrt{x^{2}-8 x+40} .
$$

It easy see that $T$ is a continuous uniformly $L$-Lipschitzian and a total asymptotically non-expansive mapping with $F(T)=\{5\}$.

Let $\alpha_{n}=\beta_{n}=\frac{n}{n+1}$ and $\gamma_{n}=1$ for all $n \in \mathbb{N}$ and without perturbations. By using Wolfram Mahtematica 10, we computed the iterates of (4), (6)-(8) (and moreover (8) with $\alpha_{n}=0$, hybrid Siteration) for an initial point $x_{1}=999$. The convergence behaviour of all iterations for approximating the fixed point 5 are given in Figures 1 and 2.

In Figures 3, and 4, we give the convergence behaviour of the iterates of and for some initial point under the different control conditions.

Example 3.2: Consider $X=\mathbb{R}^{2}$ equipped with the Euclidean norm. Let $x=\left(x_{1}, x_{2}\right) \in \mathbb{R}^{2}$, then the squared distance of $x$ from the origin is

$$
\|x\|^{2}=x_{1}^{2}+x_{2}^{2}
$$

Consider $K$ as the closed unit disk:

$$
K=\left\{\left(x_{1}, x_{2}\right) \in \mathbb{R}^{2}: x_{1}^{2}+x_{2}^{2} \leq 1\right\},
$$

which is bounded, closed, and convex in $X$. We define mapping $\operatorname{Rot}_{\theta}: K \rightarrow K$ by:

$$
\operatorname{Rot}_{\theta}\left(x_{1}, x_{2}\right)=\left[\begin{array}{cc}
\cos (\theta) & -\sin (\theta) \\
\sin (\theta) & \cos (\theta)
\end{array}\right]\left[\begin{array}{l}
x_{1} \\
x_{2}
\end{array}\right] .
$$




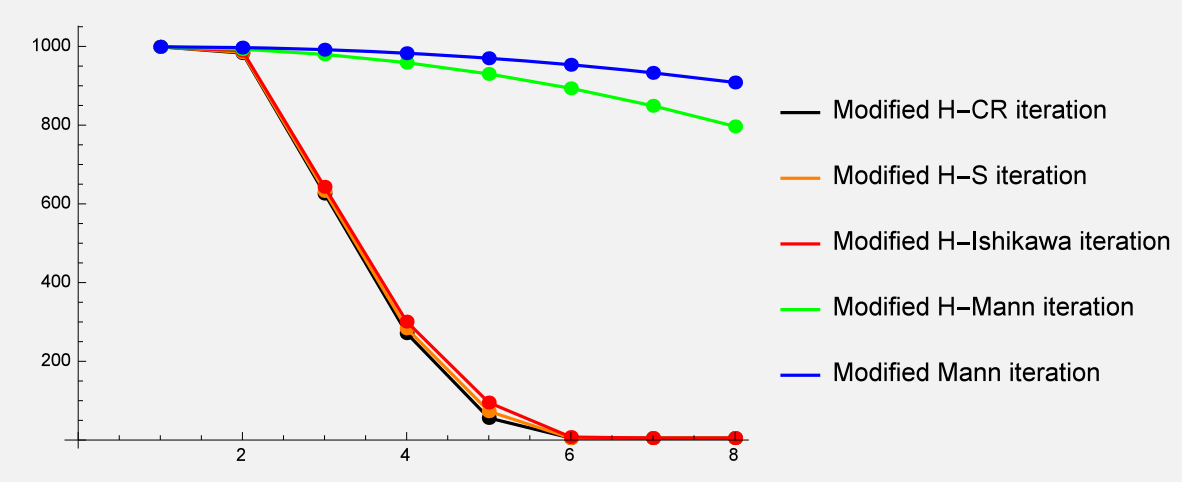

Figure 1. Convergence behaviour for Ex. 1 in case $\alpha_{n}=\beta_{n}=\frac{n}{n+1}$ and $\gamma_{n}=1$ for all $n \in \mathbb{N}$.

\begin{tabular}{|lccccc|}
\hline \multicolumn{7}{c}{ Iterate } & Mann & H-Mann & H-Ishikawa & H-S & H-CR \\
\hline$x_{1}$ & 999. & 999. & 999. & 999. & 999. \\
$x_{2}$ & 997.006 & 993.018 & 989.03 & 985.042 & 983.049 \\
$x_{3}$ & 991.689 & 979.725 & 643.415 & 632.765 & 626.158 \\
$x_{4}$ & 982.716 & 958.791 & 300.868 & 283.686 & 271.47 \\
$x_{5}$ & 969.956 & 930.082 & 95.324 & 73.1034 & 56.1376 \\
$x_{6}$ & 953.341 & 893.537 & 7.66672 & 5.00009 & 5. \\
$x_{7}$ & 932.836 & 849.119 & 5.00023 & 5 & 5. \\
$x_{8}$ & 908.416 & 796.811 & 5.00006 & 5 & 5. \\
\hline
\end{tabular}

Figure 2. Convergence behaviour for Example 3.1 in case $\alpha_{n}=\beta_{n}=\frac{n}{n+1}$ and $\gamma_{n}=1$ for all $n \in \mathbb{N}$.

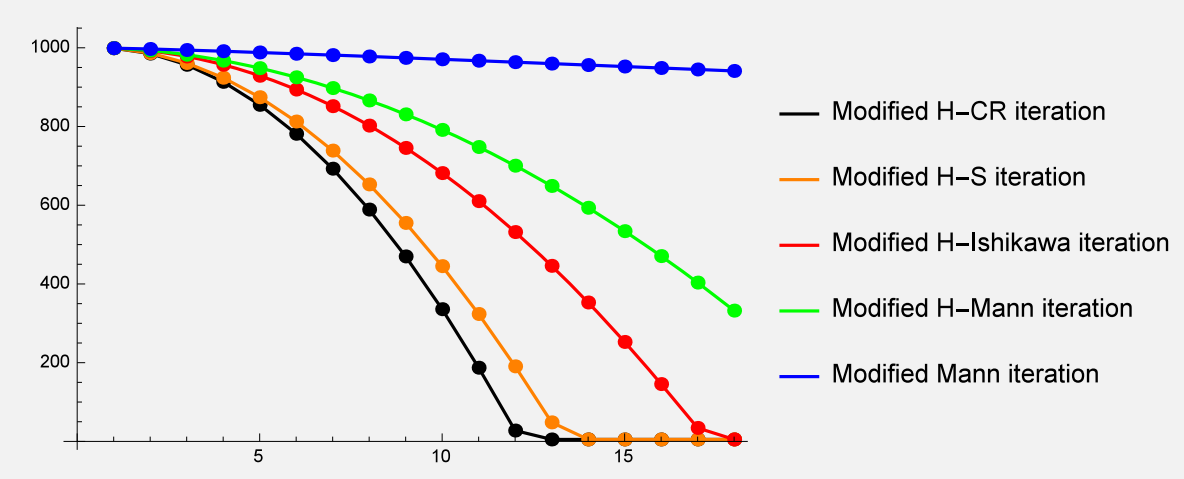

Figure 3. Convergence behaviour for Example 3.1 in case $\alpha_{n}=\beta_{n}=1-\frac{1}{\sqrt{n+1}}$ and $\gamma_{n}=\frac{1}{n+1}$ for all $n \in \mathbb{N}$.

For $\theta=\frac{\pi}{4}$, our algorithm is the following:

$$
\begin{aligned}
x_{(n+1)} & =\left(\begin{array}{cc}
2^{-\frac{n}{2}} & 2^{-\frac{n}{2}} \\
(-1)^{n} 2^{-\frac{n}{2}} & 2^{-\frac{n}{2}}
\end{array}\right) w_{(n)} \\
w_{(n)} & =\left(1-\alpha_{n}\right) y_{(n)}+\alpha_{n}\left(\begin{array}{cc}
2^{-\frac{n}{2}} & 2^{-\frac{n}{2}} \\
(-1)^{n} 2^{-\frac{n}{2}} & 2^{-\frac{n}{2}}
\end{array}\right) y_{(n)}
\end{aligned}
$$




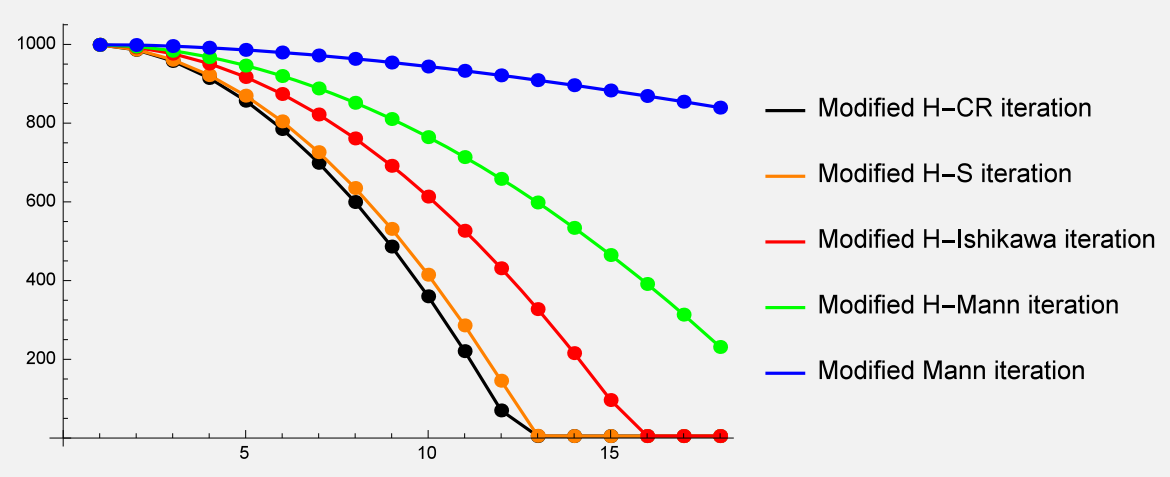

Figure 4. Convergence behaviour for Example 3.1 in case $\alpha_{n}=\beta_{n}=\frac{1}{\sqrt{n+1}}-\frac{1}{n \sqrt{n+2}}$ and $\gamma_{n}=\frac{n}{n+1}$ for all $n \in \mathbb{N}$.

$$
\begin{aligned}
& y_{(n)}=\left(1-\beta_{n}\right)\left(\begin{array}{cc}
2^{-\frac{n}{2}} & 2^{-\frac{n}{2}} \\
(-1)^{n} 2^{-\frac{n}{2}} & 2^{-\frac{n}{2}}
\end{array}\right) x_{(n)}+\beta_{n}\left(\begin{array}{cc}
2^{-\frac{n}{2}} & 2^{-\frac{n}{2}} \\
(-1)^{n} 2^{-\frac{n}{2}} & 2^{-\frac{n}{2}}
\end{array}\right) z_{(n)} \\
& z_{(n)}=\left(1-\gamma_{n}\right) x_{(n)}+\gamma_{n}\left(\begin{array}{cc}
2^{-\frac{n}{2}} & 2^{-\frac{n}{2}} \\
(-1)^{n} 2^{-\frac{n}{2}} & 2^{-\frac{n}{2}}
\end{array}\right) x_{(n)} .
\end{aligned}
$$

It is easy to see that $\operatorname{Rot}_{\theta}$ is non-expansive, since for all $\left(x_{1}, x_{2}\right),\left(y_{1}, y_{2}\right) \in K$ and $\mathrm{R}_{\theta}$ is total asymptotically non-expansive mapping. Clearly, zero is the only fixed point of the mapping $\operatorname{Rot}_{\theta}$.

Let $\alpha_{n}=1-\frac{1}{\sqrt{n+1}}$ and $\beta_{n}=\gamma_{n}=\frac{1}{n+1}$ for all $n \in \mathbb{N}$ and without perturbations. By using Wolfram Mahtematica 10, we computed the iterates of (8) for an initial point $x_{(1)}=(250,250)$ for $\theta=\frac{\pi}{4}, \frac{\pi}{8}$ and $\frac{\pi}{14}$. The convergence behaviour of all iterations for approximating the fixed point $(0,0)$ are given in Figures 5 and 6.

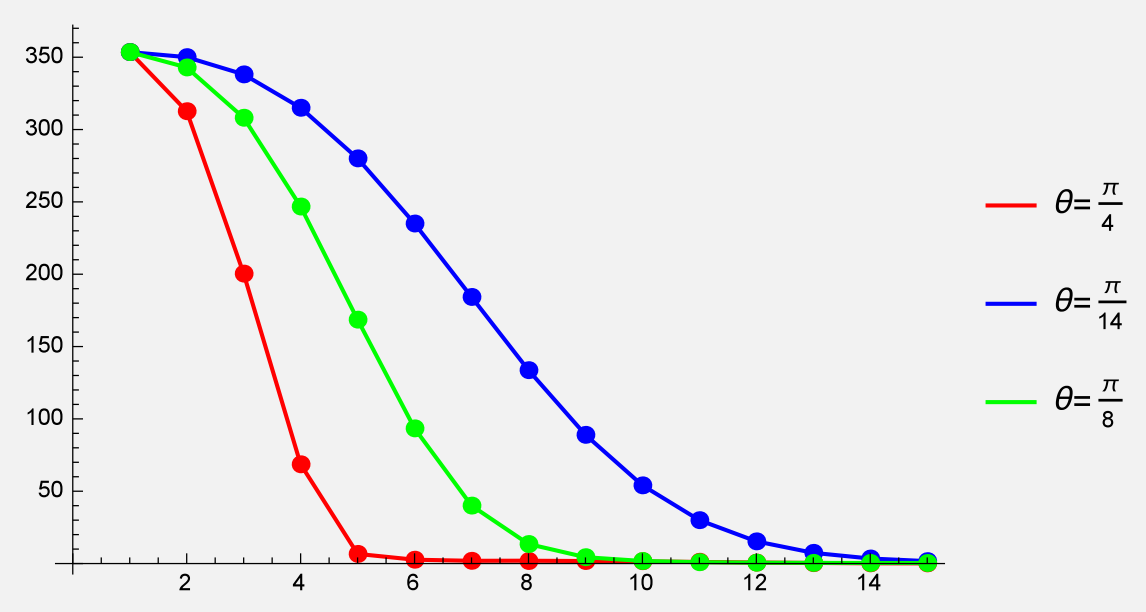

Figure 5. Convergence behaviour for Ex. 2. 


\begin{tabular}{lccc}
\hline Error & $\theta=\frac{\pi}{4}$ & $\theta=\frac{\pi}{8}$ & $\theta=\frac{\pi}{14}$ \\
\hline$\left\|x_{(1)}^{2}\right\|$ & 353.553 & 353.553 & 353.553 \\
$\left\|x_{(2)}^{2}\right\|$ & 312.684 & 342.933 & 350.055 \\
$\left\|x_{(3)}^{2}\right\|$ & 200.42 & 308.158 & 338.135 \\
$\left\|x_{(4)}^{2}\right\|$ & 68.5979 & 246.782 & 315.054 \\
$\left\|x_{(5)}^{2}\right\|$ & 6.66271 & 168.598 & 280.081 \\
$\left\|x_{(6)}^{2}\right\|$ & 2.65704 & 93.4389 & 235.044 \\
$\left\|x_{(7)}^{2}\right\|$ & 1.8949 & 40.062 & 184.281 \\
$\left\|x_{(8)}^{2}\right\|$ & 1.75554 & 13.4935 & 133.676 \\
$\left\|x_{(9)}^{2}\right\|$ & 1.75554 & 4.38678 & 88.9765 \\
$\left\|x_{(10)}^{2}\right\|$ & 1.63583 & 1.76351 & 54.0581 \\
$\left\|x_{(11)}^{2}\right\|$ & 1.23427 & 0.921586 & 29.9896 \\
$\left\|x_{(12)}^{2}\right\|$ & 0.666829 & 0.600032 & 15.3399 \\
$\left\|x_{(13)}^{2}\right\|$ & 0.293425 & 0.461712 & 7.39926 \\
$\left\|x_{(14)}^{2}\right\|$ & 0.167443 & 0.400777 & 3.48589 \\
$\left\|x_{(15)}^{2}\right\|$ & 0.130935 & 0.377136 & 1.6708 \\
\end{tabular}

Figure 6. Convergence behaviour for Ex. 2.

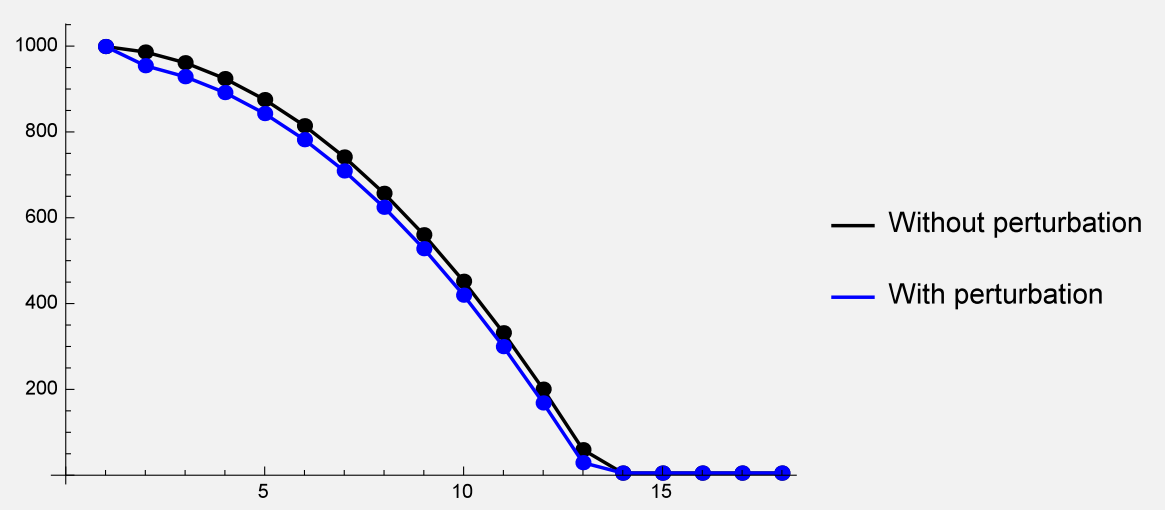

Figure 7. Convergence behaviour for Example 3.3.

Example 3.3: Here we reconsider Example 3.1, in case $\alpha_{n}=\beta_{n}=\frac{1}{\sqrt{n+1}}-\frac{1}{\sqrt{n+2}}$ and $\gamma_{n}=\frac{n}{n+1}$ for all $n \in \mathbb{N}$ (see Figure 9), where we consider a version with perturbation given by $\alpha_{n}^{\prime}=\beta_{n}^{\prime}=\gamma_{n}^{\prime}=n^{-5}$ and $\epsilon_{n}=\epsilon_{n}^{\prime}=\epsilon_{n}^{\prime \prime}=\frac{(n+1) n^{3}}{2^{n}}-16$ (Figures 7 and 8 ).

Example 3.4: Here we reconsider Example 3.2, with same $\alpha_{n}=1-\frac{1}{\sqrt{n+1}}$ and $\beta_{n}=\gamma_{n}=\frac{1}{n+1}$ for all $n \in \mathbb{N}$ and with perturbations given by $\alpha_{n}^{\prime}=\beta_{n}^{\prime}=\gamma_{n}^{\prime}=n^{-5}$ and $\epsilon_{n}=\epsilon_{n}^{\prime}=\epsilon_{n}^{\prime \prime}=\frac{(n+1) n^{3}}{2^{n}}-16$. By using Wolfram Mahtematica 10, we computed the iterates of (8) for an initial point $x_{(1)}=(250,250)$ for $\theta=\frac{\pi}{4}, \frac{\pi}{8}$ and $\frac{\pi}{14}$. The convergence behaviour of all iterations for approximating the fixed point $(0,0)$ are given in Figure 9, where we compare pertubed and no perturbed ones. 


\begin{tabular}{|lcc|}
\hline & & \\
\hline Iterate & Hybrid CR & Perturbed Hybrid CR \\
\hline$x_{1}$ & 999. & 999. \\
$x_{2}$ & 986.26 & 954.314 \\
$x_{3}$ & 961.306 & 928.714 \\
$x_{4}$ & 924.316 & 891.707 \\
$\vdots$ & $\vdots$ & $\vdots$ \\
$x_{11}$ & 332.015 & 299.626 \\
$x_{12}$ & 200.602 & 168.44 \\
$x_{13}$ & 59.5073 & 29.2372 \\
$x_{14}$ & 5. & 5. \\
\hline
\end{tabular}

Figure 8. Convergence behaviour for Example 3.3.

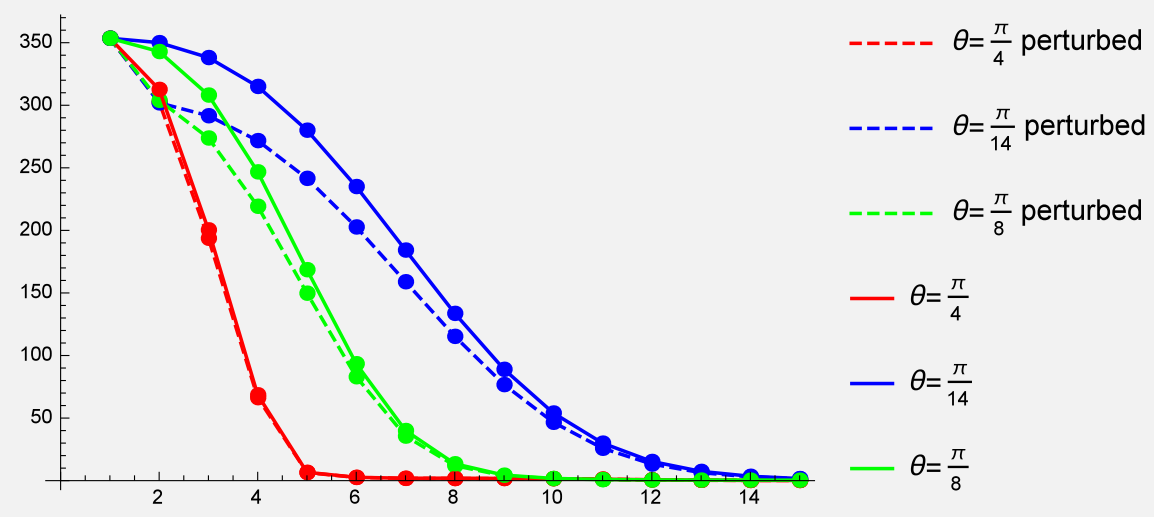

Figure 9. Convergence behaviour for Example 3.4.

\section{Disclosure statement}

No potential conflict of interest was reported by the authors.

\section{ORCID}

Kenyi Calderón (D) http://orcid.org/0000-0001-5361-3351

J. Martínez-Moreno (D) http://orcid.org/0000-0002-3340-2781

E. M. Rojas (D) http://orcid.org/0000-0002-8593-0463

\section{References}

[1] R.P. Agarwal, D.O Regan, and D.R. Sahu, Iterative construction of fixed points of nearly asymptotically nonexpansive mappings, J. Nonlinear Convex Anal. 8 (2007), pp. 61-79.

[2] Y. Alber, R. Espinola, and P. Lorenzo, Strongly convergent approximations to fixed points of total asymptotically nonexpansive mappings, Acta Mech. Sin. 24 (2008), pp. 1005-1022.

[3] S.S. Chang, L. Wang, H.J. Lee, C.K. Chan, and L. Yang, Demiclosed principle and $\Delta$-convergence theorems for total asymptotically nonexpansive mappings in CAT(0) spaces, Appl. Math. Comput. 219 (2012), pp. 2611-2617.

[4] R. Chugh, V. Kumar, and S. Kumar, Strong convergence of a new three step Iterative scheme in Banach spaces, Am. J. Comput. Math. 2 (2012), pp. 345-357.

[5] S. Dhompongsa, W.A. Kirk, and B. Panyanak, Nonexpansive set-valued mappings in metric and Banach spaces, J. Nonlinear Convex Anal. 8 (2007), pp. 35-45.

[6] S. Dhompongsa and B. Panyanak, On $\Delta$-convergence theorems in CAT(0) spaces, Comput. Math. Appl. 56 (2008), pp. 2572-2579. 
[7] N. Hussain and M.A. Khamsi, On asymptotic pointwise contractions in metric spaces, Nonlinear Anal. 71 (2009), pp. 4423-4429.

[8] W.A. Kirk and B. Panyanak, A concept of convergence in geodesic spaces, Nonlinear Anal. 68 (2008), pp. $3689-3696$.

[9] W. Kumam, N. Pakkaranang, and P. Kumam, Modified viscosity type iteration for total asymptotically nonexpansive mappings in CAT(0) spaces and its application to optimization problems, J. Nonlinear Sci. Appl. 11 (2018), pp. 288-302.

[10] W. Kumam, N. Pakkaranang, P. Kumam, and P. Cholamjiak, Convergence analysis of modified Picard-S hybrid iterative algorithms for total asymptotically nonexpansive mappings in Hadamard spaces, (Published online) to appear in Int. J. Comput. Math. (2018). doi:10.1080/00207160.2018.1476685.

[11] N. Pakkaranang, P. Kumam, and Y.J. Cho, Proximal point algorithms for solving convex minimization problem and common fixed points problem of asymptotically quasi-nonexpansive mappings in CAT(0) spaces with convergence analysis, Numer. Algorithms 78 (2018), pp. 827-845.

[12] N. Pakkaranang, P. Kumam, Y.J. Cho, P. Saipara, A. Padcharoen, and C. Khaofong, Strong convergence of modified viscosity implicit approximation methods for asymptotically nonexpansive mappings in complete CAT( 0$)$ spaces, J. Math. Comput. Sci. 17 (2017), pp. 345-354.

[13] A. Pansuwan and W. Sintunavarat, A new iterative scheme for numerical reckoning fixed points of total asymptotically nonexpansive mappings, Fixed Point Theory Appl. 2016 (2016), pp. 83.

[14] X. Qin, S. Cho, and S. Kang, A weak convergence theorem for total asymptotically pseudocontractive mappings in Hilbert spaces, Fixed Point Theory Appl. 1 (2011), pp. 859795.

[15] G.S. Saluja and M. Postolache, Three-step iterations for total asymptotically nonexpansive mappings in CAT(0) spaces, Filomat 31 (2017), pp. 1317-1330.

[16] J. Schu, Weak and strong convergence to fixed points of asymptotically nonexpansive mappings, Bull. Aust. Math. Soc. 43 (1991), pp. 153-159.

[17] K.K. Tan and H.K. Xu, Approximating fixed points of non-expansive mappings by the Ishikawa iteration process, J. Math. Anal. Appl. 178 (1993), pp. 301-301.

[18] K.K. Tan and H.K. Xu, Fixed point iteration processes for asymptotically nonexpansive mappings, Proc. Amer. Math. Soc. 122 (1994), pp. 733-739.

[19] B.S. Thakur, D. Thakur, and M. Postolache, Modified Picard-Mann hybrid iteration process for total asymptotically nonexpansive mappings, Fixed Point Theory Appl. 2015 (2015), pp. 140.

[20] I. Uddin, J.J. Nieto, and J. Ali, One-step iteration scheme for multivalued nonexpansive mappings in CAT(0) spaces, Mediterr. J. Math. 13 (2016), pp. 1211-1225. 